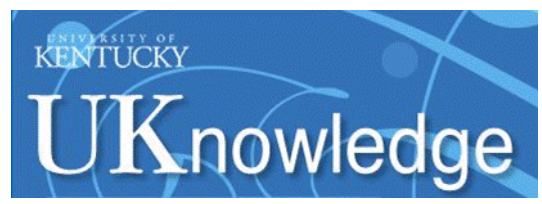

University of Kentucky

UKnowledge

9-15-2016

\title{
High-Velocity Bipolar Molecular Emission from an AGN Torus
}

\author{
Jack F. Gallimore \\ Bucknell University \\ Moshe Elitzur \\ University of Kentucky, moshe@pa.uky.edu \\ Robert Maiolino \\ University of Cambridge, UK \\ Alessandro Marconi \\ Università di Firenze, Italy \\ Christopher P. O'Dea \\ University of Manitoba, Canada
}

See next page for additional authors

Follow this and additional works at: https://uknowledge.uky.edu/physastron_facpub

Part of the Astrophysics and Astronomy Commons, and the Physics Commons

Right click to open a feedback form in a new tab to let us know how this document benefits you.

\section{Repository Citation}

Gallimore, Jack F.; Elitzur, Moshe; Maiolino, Robert; Marconi, Alessandro; O'Dea, Christopher P.; Lutz, Dieter; Baum, Stefi A.; Nikutta, Robert; Impellizzeri, C. M. V.; Davies, Richard; Kimball, Amy E.; and Sani, Eleonora, "High-Velocity Bipolar Molecular Emission from an AGN Torus" (2016). Physics and Astronomy Faculty Publications. 416.

https://uknowledge.uky.edu/physastron_facpub/416

This Article is brought to you for free and open access by the Physics and Astronomy at UKnowledge. It has been accepted for inclusion in Physics and Astronomy Faculty Publications by an authorized administrator of UKnowledge. For more information, please contact UKnowledge@lsv.uky.edu. 


\section{High-Velocity Bipolar Molecular Emission from an AGN Torus}

\section{Digital Object Identifier (DOI)}

https://doi.org/10.3847/2041-8205/829/1/L7

\section{Notes/Citation Information}

Published in The Astrophysical Journal Letters, v. 829, no. 1, L7, p. 1-5.

(c) 2016. The American Astronomical Society. All rights reserved.

The copyright holder has granted the permission for posting the article here.

\section{Authors}

Jack F. Gallimore, Moshe Elitzur, Robert Maiolino, Alessandro Marconi, Christopher P. O'Dea, Dieter Lutz, Stefi A. Baum, Robert Nikutta, C. M. V. Impellizzeri, Richard Davies, Amy E. Kimball, and Eleonora Sani 


\title{
HIGH-VELOCITY BIPOLAR MOLECULAR EMISSION FROM AN AGN TORUS
}

\author{
Jack F. Gallimore ${ }^{1}$, Moshe Elitzur ${ }^{2,3}$, Roberto Maiolino ${ }^{4,5}$, Alessandro Marconi ${ }^{6,7}$, Christopher P. O’'Dea ${ }^{8}$, \\ Dieter Lutz ${ }^{9}$, Stefi A. Baum ${ }^{8}$, Robert Nikutta ${ }^{10}$, C. M. V. Impellizzeri ${ }^{11}$, Richard Davies ${ }^{9}$, \\ Amy E. Kimball ${ }^{12}$, AND EleOnORA SAni ${ }^{13}$ \\ ${ }^{1}$ Dept. of Physics \& Astronomy, Bucknell University, Lewisburg, PA 17837, USA; jgallimo@bucknell.edu \\ ${ }^{2}$ Astronomy Dept., University of California, Berkeley, CA 94720, USA \\ ${ }^{3}$ Physics \& Astronomy, University of Kentucky, Lexington, KY 40506, USA \\ ${ }_{5}^{4}$ Cavendish Laboratory, University of Cambridge, 19 J. J. Thomson Avenue, Cambridge CB3 OHE, UK \\ ${ }^{5}$ Kavli Institute for Cosmology, University of Cambridge, Madingley Road, Cambridge CB3 OHA, UK \\ ${ }^{6}$ Dipartimento di Fisica e Astronomia, Università di Firenze, via G. Sansone 1, I-50019 Sesto Fiorentino (Firenze), Italy \\ 7 INAF-Osservatorio Astrofisico di Arcetri, Largo E. Fermi 5, I-50125 Firenze, Italy \\ ${ }^{8}$ University of Manitoba, Department of Physics and Astronomy, Winnipeg, MB R3T 2N2, Canada \\ ${ }^{9}$ Max Planck Institute for Extraterrestrial Physics, Giessenbachstrasse 1, D-85748 Garching, Germany \\ ${ }^{10}$ Instituto de Astrofísica, Facultad de Física, Pontificia Universidad Católica de Chile, 306, Santiago 22, Chile \\ ${ }^{11}$ Joint ALMA Office, Alsonso de Cordova 3107, Vitacura, Santiago, Chile \\ ${ }^{12}$ National Radio Astronomy Observatory, 1003 Lopezville Road, Socorro, NM 87801, USA \\ ${ }^{13}$ European Southern Observatory, Alonso de Cordova 3107, Vitacura, Santiago, Chile \\ Received 2016 July 21; revised 2016 August 2; accepted 2016 August 7; published 2016 September 15
}

\begin{abstract}
We have detected in ALMA observations CO $J=6 \rightarrow 5$ emission from the nucleus of the Seyfert galaxy NGC 1068. The low-velocity (up to $\pm 70 \mathrm{~km} \mathrm{~s}^{-1}$ relative to systemic) CO emission resolves into a $12 \times 7 \mathrm{pc}$ structure, roughly aligned with the nuclear radio source. Higher-velocity emission (up to $\pm 400 \mathrm{~km} \mathrm{~s}^{-1}$ ) is consistent with a bipolar outflow in a direction nearly perpendicular $\left(\simeq 80^{\circ}\right)$ to the nuclear disk. The positionvelocity diagram shows that in addition to the outflow, the velocity field may also contain rotation about the disk axis. These observations provide compelling evidence in support of the disk-wind scenario for the active galactic nucleus obscuring torus.
\end{abstract}

Key words: galaxies: active - galaxies: individual (NGC 1068) - galaxies: nuclei - galaxies: Seyfert quasars: general

\section{INTRODUCTION}

The great diversity of active galactic nucleus (AGN) classes has been explained by a single unified scheme. The nuclear activity is powered by accretion onto a supermassive $\left(\sim 10^{6}-\right.$ $10^{10} M_{\odot}$ ) black hole (BH). The active region is surrounded by an obscuring, dusty toroidal structure so that sources viewed face-on are recognized as type 1 AGNs, and those observed edge-on are type 2 (e.g., Antonucci 1993; Urry \& Padovani 1995). However, the origin and nature of the obscuring torus remain far from understood, with proposed models broadly divided into two main classes. In one, the torus is a stationary doughnut-like structure (Krolik \& Begelman 1988), and its large dimensions ( $\gtrsim 100 \mathrm{pc}$ ) place it well outside the $\mathrm{BH}$ radius of gravitational influence (Granato \& Danese 1994). In the other, a dynamic picture is employed: the torus is made of gas processed by the accretion disk and expelled outward in a disk wind; the outflow inner part is atomic and ionized, giving rise to broad-line emission, its outer regions are molecular and dusty, resulting in a compact (well within the $\mathrm{BH}$ gravitational potential), axisymmetric obscuring structure that mimics a hydrostatic toroidal distribution (Elitzur \& Shlosman 2006 and references therein).

Beginning with the VLTI detection of the nucleus in NGC 1068 (Jaffe et al. 2004), infrared (IR) observations have established the compact dimensions of AGN dusty tori (Elitzur 2008; Nenkova et al. 2008). Optical-near-IR reverberation lags in Type 1 Seyferts are consistent with sub-parsec torus inner radii (e.g., Vazquez et al. 2015 and references therein). Further confirmation comes from recent ALMA observations by García-Burillo (2016, hereafter GB16). They detected molecular emission from the NGC 1068 torus, contained within $\sim 3 \mathrm{pc}$ from the center. However, although the torus's compact dimensions are now firmly established, its kinematics and dynamic origin remain uncertain. Water maser observations provide support for the disk-wind scenario in Circinus (Greenhill et al. 2003) and NGC 3079 (Kondratko et al. 2005), but the tight constraints on strong maser emission do not allow a complete sampling of the molecular gas.

Here, we report our own ALMA observations of thermal CO emission from the NGC 1068 torus. We adopt a distance to the source of 14.4 Mpc (Bland-Hawthorn et al. 1997). The scale is $1^{\prime \prime}=70 \mathrm{pc}$.

\section{OBSERVATIONS AND DATA PROCESSING}

We observed NGC 1068 in Band $9(\lambda \sim 450 \mu \mathrm{m})$ during ALMA Cycle 2 (project code 2013.1.00014.S). Total time on source was 32.1 minutes. The baseline range was $15-1466 \mathrm{~m}$, and the synthetic restoring beam (angular resolution) is 0 " $084 \times 0$ " 064 , PA $75^{\circ}$, or $5.9 \times 4.5 \mathrm{pc}$. Resulting images are insensitive to structures larger than about $270 \mathrm{pc}(3 . ! 8)$. Source observations were interleaved with short scans of the nearby bright calibrator source J0217+0144, located 6.5 from NGC 1068. Spectral windows were tuned to observe three independent continuum bands, $2 \mathrm{GHz}$ bandwidth each, and one line window for $\mathrm{CO}\left(J=6 \rightarrow 5 ; \nu_{0}=691.47308 \mathrm{GHz}\right.$, hereafter $\mathrm{CO})$ in $\sim 0.21 \mathrm{~km} \mathrm{~s}^{-1}$ channels spanning $\sim \pm 400 \mathrm{~km} \mathrm{~s}^{-1}$ total bandwidth. Calibration and data reduction followed standard procedures for ALMA observations in CASA (CASA v.4.3.1; McMullin et al. 2007). NGC 1068 proved bright enough for self-calibration over scan intervals. 
Two rounds of phase-only self-calibration reduced sidelobe artifacts and improved the background rms.

The photometric accuracy, $\sim 10 \%$, is limited by the photometric uncertainties of the flux calibrators (3C454.3 and J2253+1608). The absolute astrometric accuracy of these ALMA observations are limited by the transfer of calibration solutions from the complex gain calibrators to NGC 1068. We estimate that the final absolute astrometric accuracy ${ }^{14}$ of our observations is $1.3 \mathrm{pc}$ (19 mas). This systematic uncertainty is large compared to the $\sim 4 \mathrm{pc}$ scale of the nuclear environment. We therefore adopted an astrometric correction by aligning the Band 9 continuum peak to the position of the active nucleus (cf. GB16). In angular units, the shift moves the Band 9 continuum peak 9.5 mas west and 11 mas south. In physical units, the shift is $1.0 \mathrm{pc}$, which lies within the $1.3 \mathrm{pc}$ systematic uncertainty.

Images and data cubes were produced using Briggs weighting (Briggs 1995) and multiscale CLEAN deconvolution (Cornwell 2008). The CO spectral line cube was binned to $10 \mathrm{~km} \mathrm{~s}^{-1}$ channel-widths during imaging and deconvolution. The background rms noise levels are $0.49 \mathrm{mJy} \mathrm{beam}^{-1}$ and $5.3 \mathrm{mJy}^{\text {beam }}{ }^{-1}$ for Band 9 continuum and the $\mathrm{CO}$ channel maps, respectively.

\section{RESULTS}

GB16 performed similar observations of NGC 1068, and their continuum and integrated line maps resemble ours. Like GB16, we detect continuum and molecular emission located at the position of the nuclear radio source, S1, and its associated $\mathrm{H}_{2} \mathrm{O}$ vapor maser disk (Greenhill \& Gwinn 1997; Gallimore et al. 2004). The detected CO spans the full $\pm 400 \mathrm{~km} \mathrm{~s}^{-1}$ spectral range. Figure 1 shows the $\mathrm{CO}$ image of S1 integrated over three bins: blueshifted, $v<-70 \mathrm{~km} \mathrm{~s}^{-1}$ (blue contours); systemic, $\quad-70 \leqslant v \leqslant$ $+70 \mathrm{~km} \mathrm{~s}^{-1}$ (grayscale); and redshifted, $v>+70 \mathrm{~km} \mathrm{~s}^{-1}$ (red contours), where $v$ is the observed radial velocity relative to systemic. The choice of $\pm 70 \mathrm{~km} \mathrm{~s}^{-1}$ for velocity bin edges was based on initial inspection of the data and the analysis of nearinfrared $\mathrm{H}_{2}$ emission (Galliano \& Alloin 2002; Müller Sánchez et al. 2009). At low $v$, the source resolves along PA $112^{\circ}$ (green dashed line), extending $\sim 10 \mathrm{pc}$ southeast of the VLBA source.

The CO source is unresolved in the integrated blueshifted and redshifted velocity bins. However, the blue contours are systematically displaced with respect to the red ones along the dashed black axis in Figure 1; in particular, the position of the blueshifted velocity peak is displaced $1.2 \mathrm{pc}$ northeast $\left(\mathrm{PA} \simeq 33^{\circ}\right)$ of the redshifted peak. The displacement axis is roughly orthogonal $\left(78^{\circ} \pm 2^{\circ}\right)$ to the line of masers, which trace a nearly edge-on disk. Thus, the high-velocity $C O$ emission is consistent with a bipolar outflow along the AGN axis, similar to the outflow inferred from observations of ionized gas in the narrow-line region of the NGC 1068 nucleus (Crenshaw \& Kraemer 2000).

Additional evidence for the $\mathrm{CO}$ bipolar outflow comes from the spectral map in the left panel of Figure 2. Here, we show the locations of $\mathrm{CO}$ emission peaks from the full spectral line cube. We performed spectroastrometry using find_peaks from the photutils package of Astropy (Astropy Collaboration et al. 2013). Peaks $>3.0 \sigma$ on individual channel maps were

\footnotetext{
${ }^{14}$ Based on information provided by the ALMA Helpdesk.
}

recorded, and their positions were refined using a local 2D Gaussian fit. The map shows that each high-velocity bin predominates in one of the two hemispheres defined by the maser disk, as expected from a bipolar outflow roughly aligned with the inner part of the radio jet, whose contours are also shown. We propose that the $\mathrm{CO}$ emission arises from a bipolar outflow around the axis of the AGN accretion disk. Molecular gas moving in the plane of the sky contributes to the systemicvelocity bin, whereas gas on the near (far) side of the plane appears in the blue (red) bin.

The outflow axis of ionized gas traced by optical emission lines in the narrow-line region is inclined $5^{\circ}$ out of the plane of the sky (Crenshaw \& Kraemer 2000), and we assume the same for the $\mathrm{CO}$ outflow. Can such a small inclination produce the red-blue spatial separation observed in $\mathrm{CO}$ ? To test this possibility we conducted a numerical simulation of a bipolar outflow. We employ a truncated cone geometry with a disk base diameter of $1 \mathrm{pc}$ (comparable to the $\mathrm{H}_{2} \mathrm{O}$ maser disk), $4.4 \mathrm{pc}$ total bicone height (based on the spread of $\mathrm{CO}$ features in Figure 2), and half-opening angle of $30^{\circ}$. To model the outflow, we randomly placed a total of $10^{4}$ clumps inside the cone. Clumps were evenly distributed in cylindrical radius $\rho$ (i.e., radius projected onto the disk plane) and distance along the polar axis. The direction of motion for a clump is vertical for clumps directly above and below the disk (i.e., clumps with $\rho \leqslant 0.5 \mathrm{pc}$ ) and radial from the edge of the disk for clumps with $\rho>0.5 \mathrm{pc}$. We further assume uniform acceleration from $30 \mathrm{~km} \mathrm{~s}^{-1}$ at the molecular disk to $800 \mathrm{~km} \mathrm{~s}^{-1}$ at the cone height; these values were selected from interactive trials to give a reasonable match to the observations. Finally, all clumps are equally luminous point sources of $\mathrm{CO}$ emission, added as beam-shaped, two-dimensional Gaussians to the planes of a simulated data cube. The cone was then inclined by $5^{\circ}$, and we repeated the cube analyses on the simulated data. To simulate the effects of measurement uncertainty, each measured peak position was displaced randomly, assuming $0.5 \mathrm{pc}$ errors, comparable to the relative, observed position uncertainties. The simulation results shown in the right panel of Figure 2 broadly reproduce a parsec-scale asymmetry of redshifted and blueshifted peaks similar to that observed (left panel). This simple simulation was not meant to replicate any physical model or explain every aspect of the data; in particular, the systemicvelocity emission is concentrated to the east, at odds with the random distribution we use. Our simulation is only meant to demonstrate that the small $5^{\circ}$ tilt detected in narrow-line observations is sufficient to produce the red-blue asymmetry of the excited $\mathrm{CO}$ emission.

In addition to the outflow component described above, we also find evidence for rotation consistent with the compact $\mathrm{H}_{2} \mathrm{O}$ maser disk. Figure 3 shows the position-velocity $(p-v)$ diagram constructed by slicing the $\mathrm{CO}$ spectral line cube along the axis tracing the extended systemic emission. Surface brightnesses were averaged within the three-pixel extraction width (green dashed lines in Figure 1). The data were smoothed using a Savitzky-Golay filter (7 channels, polynomial order 3; Savitzky \& Golay 1964) along the velocity axis for display purposes. The diagram reveals $\mathrm{CO}$ emission at very high velocities, with significant $(>3 \sigma)$ detections approaching $v= \pm 400 \mathrm{~km} \mathrm{~s}^{-1}$ relative to systemic. Also shown are the $\mathrm{H}_{2} \mathrm{O}$ maser spots, appearing as a nearly vertical chain centered on the origin. Such a straight line in the $p-v$ diagram is the hallmark of a rotating ring whose angular velocity is the line 

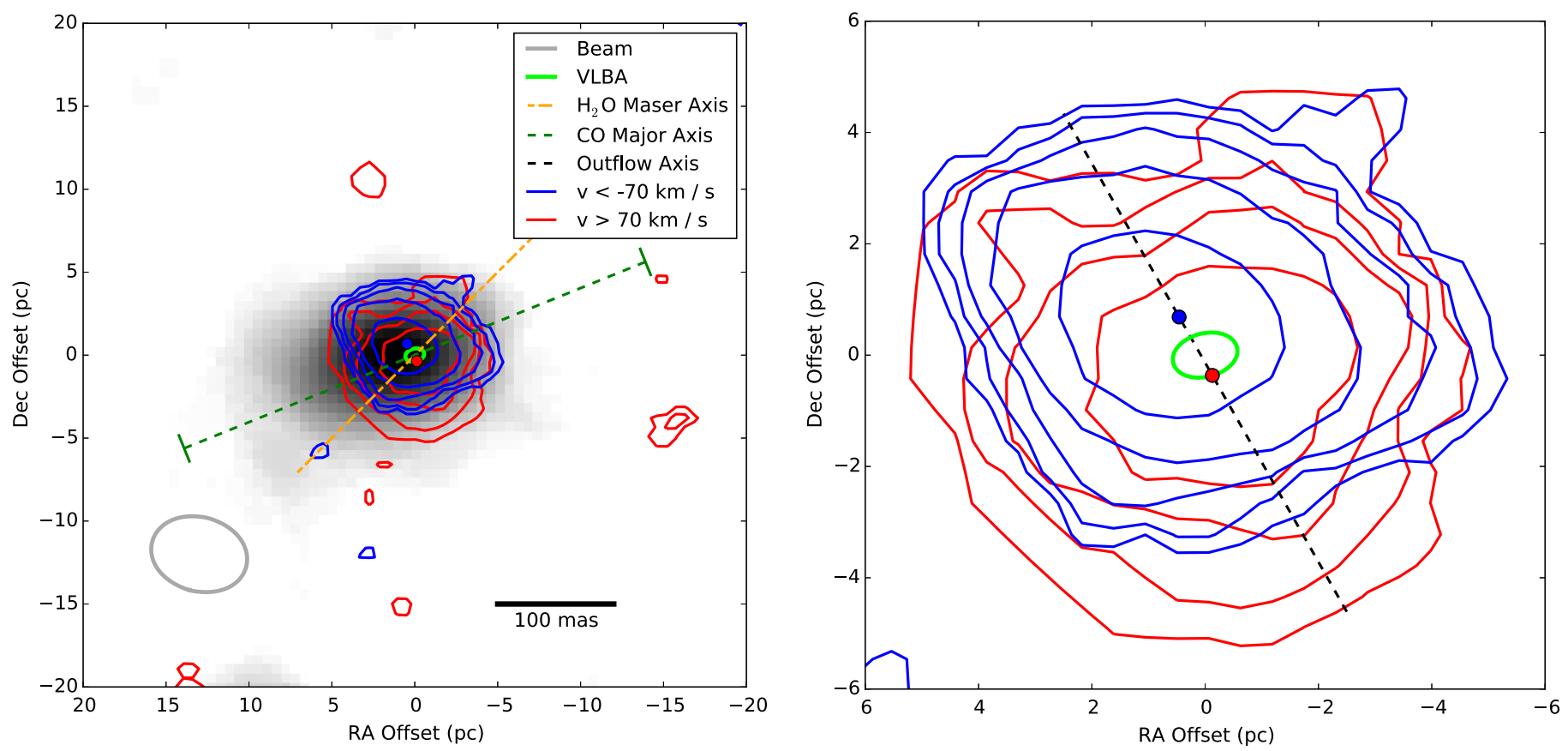

Figure 1. Left: $\mathrm{CO}$ image integrated between radial velocities -70 and $+70 \mathrm{~km} \mathrm{~s}^{-1}$ relative to systemic, shown as grayscale linearly stretched between 0.30 and $7.0 \mathrm{Jy} \mathrm{km} \mathrm{s}^{-1}$ beam $^{-1}$. Position offsets are presented in physical units and are measured with respect to the centroid of the $5 \mathrm{GHz}$ VLBA image of S1, the nuclear plasma disk whose orientation and location are illustrated by the green ellipse. The 100 mas scale bar in the lower right corner indicates the angular scale. The dashed green line (PA $112^{\circ}$ ) illustrates the CO image major axis and the extraction axis for the position-velocity diagram (see the text for details). The end-caps on the major axis line show the three-pixel ( 24 mas, or $1.8 \mathrm{pc}$ ) extraction width for the position-velocity diagram. Red and blue contours trace the integrated CO emission over velocities $>+70 \mathrm{~km} \mathrm{~s}^{-1}$ and $<-70 \mathrm{~km} \mathrm{~s}^{-1}$, respectively. The contour levels are $0.30,0.50,0.83,1.4$, and $2.3 \mathrm{Jy} \mathrm{km} \mathrm{s}^{-1}$ beam ${ }^{-1}$. The alternating dashed orange line (PA $135^{\circ}$ ) shows the orientation of the $\mathrm{H}_{2} \mathrm{O}$ maser disk, stretched beyond its $\sim 1.7 \mathrm{pc}$ major axis to emphasize its orientation at the scale of the plot. Right: zoom-in on the high-velocity contours; the contour peaks are marked by colored dots. The red and blue contours are systematically displaced from each other, with their peaks separated by $1.2 \mathrm{pc}$ along the axis PA $33^{\circ}$, which is traced by the dashed black line. This displacement is indicative of a bipolar outflow roughly orthogonal to the nuclear disk.

slope (e.g., Pestalozzi et al. 2009). The fact that the maser line is so straight and narrow implies that all maser spots are located within a radially thin annulus at the disk inner radius. The ALMA data show a similar elongated structure aligned with the maser chain, only covering a wider spread of positions. This component can be interpreted as $\mathrm{CO}$ emission from a wider range of rotating regions close to the disk innermost boundary. The thermal $\mathrm{CO}$ emission traces these molecular regions more fully than the masers because strong maser emission is not produced without the fulfillment of some strict constraints on the physical parameters. For example, these constraints likely explain the dearth of blueshifted maser spots. Additionally, the $\mathrm{CO}$ data also show another elongated component, this one closer to the horizontal axis. Its smaller slope implies a lower angular velocity, which in turn implies a larger radius and can be interpreted as emission from the outer regions of the $\mathrm{CO}$ distribution.

An elongated structure in the $p-v$ diagram could also be produced by a collimated jet, in which case the slope is directly related to the jet inclination from the line of sight (Pestalozzi et al. 2009). The existence of two such features with different slopes and the overlap of one of them with the maser line make a jet-based explanation unlikely, indicating that the $\mathrm{CO}$ velocity field contains a kinematic component consistent with rotation.

To explore this possibility we simulated the $p-v$ diagram of an edge-on disk with kinematics based on Keplerian rotation around a $1.5 \times 10^{7} M_{\odot}$ central mass that best fits the $\mathrm{H}_{2} \mathrm{O}$ maser disk observations (Lodato \& Bertin 2003) but with a line of nodes rotated $23^{\circ}$ to match the $p-v$ slice orientation. To highlight the expected contribution of such a disk, its emissivity was scaled proportional to $r^{-2}$, where $r$ is the disk radial coordinate. The simulated data were beam-smoothed and binned into $10 \mathrm{~km} \mathrm{~s}^{-1}$ channels to compare with the ALMA observations. The result is shown as transparent, violet-shaded contours in Figure 3; note the expected "tilted bow-tie" shape (cf. Pestalozzi et al. 2009).

This simulation is not intended to fit the $\mathrm{CO}$ data but to illustrate the appearance of a rotating disk on the $p-v$ diagram. The overall similarity with the data suggests the presence of rotation on top of the radial outflow, where the latter fills in the regions in the $p-v$ diagram inaccessible to rotational motion. The emerging picture is in agreement with the disk-wind model, first put forward by Blandford \& Payne (1982). Such a wind can be visualized as a stack of rotating disks that are accelerated away from the base of the outflow. Each disk in the stack could in turn be described by an analysis similar to the one that produced the violet-shaded structure in Figure 3. It thus seems that the simplest interpretation is that the $\mathrm{CO}$ emission traces a combination of rotation and radial outflow on parsec scales surrounding the central engine, consistent with the disk-wind scenario. From the Kondratko et al. (2005) observations of $\mathrm{H}_{2} \mathrm{O}$ masers in NGC 3079, Elitzur \& Ho (2009) suggest that the disk-wind launch velocity is roughly $10 \%$ of the local Keplerian velocity, or a few $\times 10 \mathrm{~km} \mathrm{~s}^{-1}$ in the case of NGC 1068. Subsequent acceleration can lead to the observed radial velocities of the order of several 

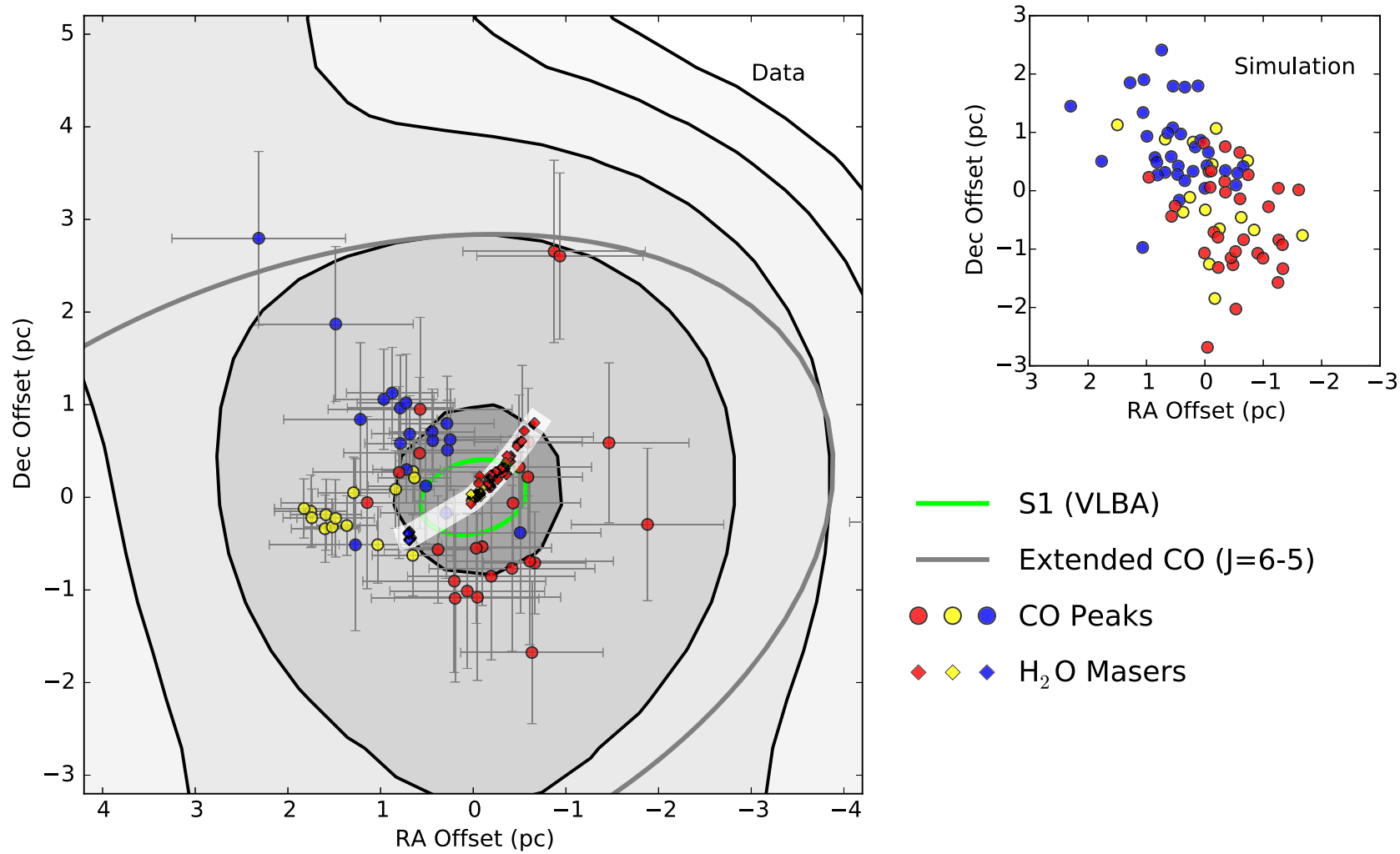

Figure 2. Left: the locations of $\mathrm{CO}$ emission peaks, marked by colored circles. The colors are coded by radial velocity bins relative to systemic: $v>70 \mathrm{~km} \mathrm{~s}{ }^{-1}$ (red), $-70 \leqslant v \leqslant 70 \mathrm{~km} \mathrm{~s}^{-1}$ (yellow), and $v<-70 \mathrm{~km} \mathrm{~s}^{-1}$ (blue). Following the same velocity color coding, the positions of $\mathrm{H}_{2} \mathrm{O}$ maser spots (Greenhill \& Gwinn 1997) are plotted as diamonds within the central, white arcuate band. The location and scale of the VLBA radio continuum source is indicated by the green ellipse. The outer gray ellipse illustrates the orientation of the resolved, low-velocity CO emission (see Figure 1). Grayscale contours are from MERLIN 5 GHz observations of the radio jet (Gallimore et al. 2004); the MERLIN beam is 4.2 pc FWHM. Upper right: spectral map from a simulation of a bipolar outflow with the axis tilted $5^{\circ}$ from the plane of the sky and rotated to PA $33^{\circ}$ (see the text for details). Colors are as for the left panel.

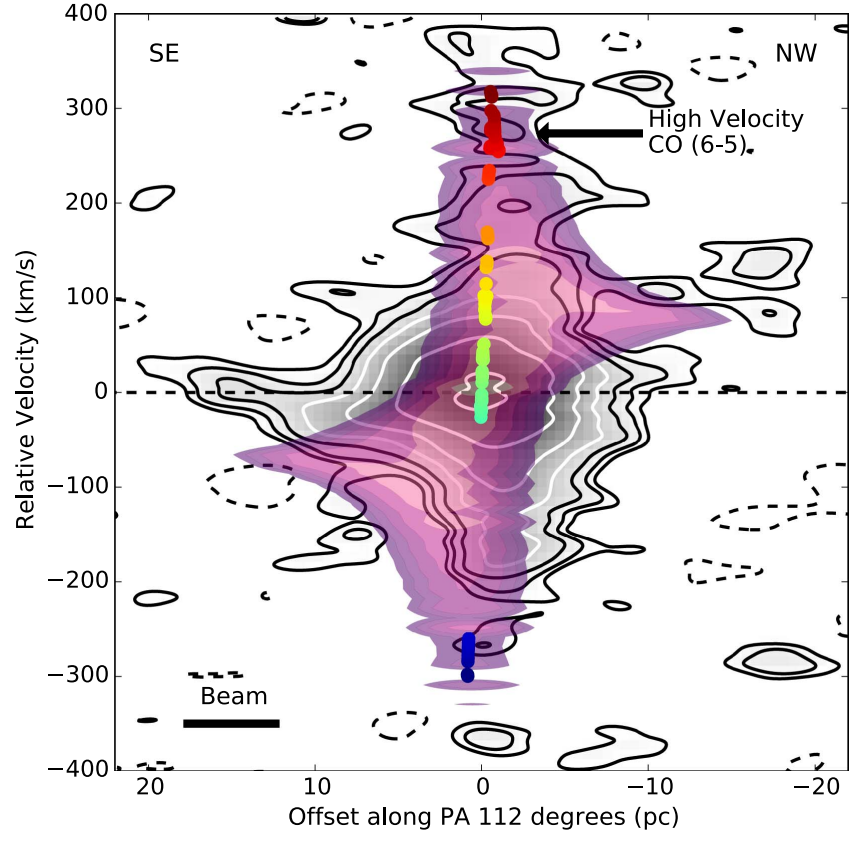

Figure 3. $\mathrm{CO}$ position-velocity diagram, with position varying along the $\mathrm{CO}$ major axis (the green dashed line at PA $112^{\circ}$ in Figure 1). The data are shown as grayscale with contours at $-4.7,4.7,7.2,11.0,16.8,25.7,39.2$, and 60 mJy beam ${ }^{-1}$. The beam width in the direction of the position slice is shown at the lower left. The rainbow-colored circles mark the $p-v$ measurements of the $\mathrm{H}_{2} \mathrm{O}$ masers. The transparent violet shading traces the emission expected from Keplerian rotation around a $1.5 \times 10^{7} M_{\odot}$ central mass (see the text for details).
$100 \mathrm{~km} \mathrm{~s}^{-1}$ (e.g., Blandford \& Payne 1982; Emmering et al. 1992; Kartje et al. 1999).

\section{DISCUSSION}

The misalignment between the maser and radio disks has been a puzzle ever since the Gallimore et al. (2004) VLBA observations. Our ALMA data show that the systemic-velocity $\mathrm{CO}$ emission is closely aligned with the $\mathrm{S} 1$ radio disk, enhancing the case for this to be the true orientation of the AGN accretion disk. The maser inclination may be attributed to the foundations of the emission process: strong maser amplification requires tight velocity coherence along the line of sight. It is possible that some transient process created favorable conditions for such coherence on the disk inner boundary at an angle to the disk plane. Either direction is nearly orthogonal to the $\mathrm{CO}$ outflow axis, which is rotated $79^{\circ}$ clockwise from the $\mathrm{S} 1$ axis and $78^{\circ}$ counterclockwise from the maser axis. Thus, the NGC 1068 nucleus has a bipolar outflow, seen in the high-velocity $\mathrm{CO}$, radio jet, and narrow optical emission lines. The outflow is roughly orthogonal to the accretion disk, which is traced by the systemic-velocity $\mathrm{CO}$, radio continuum emission, and $\mathrm{H}_{2} \mathrm{O}$ masers.

Having detected a single $\mathrm{CO}$ transition, it is difficult to derive strong constraints on the physical parameters of emitting clouds. Some indications can still be obtained from the $J=6 \rightarrow 5$ brightness temperature, which peaks at $31.1 \mathrm{~K}$. Detailed radiative transfer calculations of CO line emission show that such brightness temperature requires $\mathrm{H}_{2}$ density in 
excess of $\sim 10^{5} \mathrm{~cm}^{-3}$, temperatures higher than $\sim 50 \mathrm{~K}$, and CO column densities $N_{\mathrm{CO}} \gtrsim 10^{17} \mathrm{~cm}^{-2}\left(\mathrm{~km} \mathrm{~s}^{-1}\right)^{-1}$. From modeling of IR emission from AGNs, torus clouds are expected to have hydrogen column densities of $N_{\mathrm{H}} \sim 10^{23} \mathrm{~cm}^{-2}$, assuming the standard dust abundance $N_{\mathrm{H}}=2 \times 10^{21} A_{V} \mathrm{~cm}^{-2}$ (Nenkova et al. 2008). An intrinsic line width of $\sim 10 \mathrm{~km} \mathrm{~s}^{-1}$ would then imply a CO abundance of $\sim 10^{-5}$, similar to Galactic values.

Forthcoming publications will present detailed models and report on additional molecular and IR continuum observations of NGC 1068. The CO data reported here already provide the most compelling evidence yet in support of the disk-wind scenario for the AGN obscuring torus.

This Letter makes use of the following ALMA data: ADS/ JAO.ALMA\#2013.1.00014.S. ALMA is a partnership of ESO (representing its member states), NSF (USA) and NINS (Japan), together with NRC (Canada), NSC and ASIAA (Taiwan), and KASI (Republic of Korea), in cooperation with the Republic of Chile. The Joint ALMA Observatory is operated by ESO, AUI/NRAO and NAOJ. The National Radio Astronomy Observatory is a facility of the National Science Foundation operated under cooperative agreement by Associated Universities, Inc. This research made use of Astropy, a community-developed core Python package for Astronomy (Astropy Collaboration et al. 2013). R.N. acknowledges support by FONDECYT grant No. 3140436. R.M. acknowledges support from the UK Science and Technology Facilities Council (STFC).

Facility: ALMA.

Software: CASA, astropy.

\section{REFERENCES}

Antonucci, R. 1993, ARA\&A, 31, 473

Astropy Collaboration, Robitaille, T. P., Tollerud, E. J., et al. 2013, A\&A, 558, A33

Blandford, R. D., \& Payne, D. G. 1982, MNRAS, 199, 883

Bland-Hawthorn, J., Gallimore, J. F., Tacconi, L. J., et al. 1997, Ap\&SS, 248, 9 Briggs, D. S. 1995, PhD thesis, The New Mexico Institute of Mining and Technology

Cornwell, T. J. 2008, ISTSP, 2, 793

Crenshaw, D. M., \& Kraemer, S. B. 2000, ApJL, 532, L101

Elitzur, M. 2008, NewAR, 52, 274

Elitzur, M., \& Ho, L. C. 2009, ApJL, 701, L91

Elitzur, M., \& Shlosman, I. 2006, ApJL, 648, L101

Emmering, R. T., Blandford, R. D., \& Shlosman, I. 1992, ApJ, 385, 460

Galliano, E., \& Alloin, D. 2002, A\&A, 393, 43

Gallimore, J. F., Baum, S. A., \& O’Dea, C. P. 2004, ApJ, 613, 794

García-Burillo, S., Combes, F., Ramos Almeida, C., et al. 2016, ApJL, 823, L12

Granato, G. L., \& Danese, L. 1994, MNRAS, 268, 235

Greenhill, L. J., Booth, R. S., Ellingsen, S. P., et al. 2003, ApJ, 590, 162

Greenhill, L. J., \& Gwinn, C. R. 1997, Ap\&SS, 248, 261

Jaffe, W., Meisenheimer, K., Röttgering, H. J. A., et al. 2004, Natur, 429, 47

Kartje, J. F., Königl, A., \& Elitzur, M. 1999, ApJ, 513, 180

Kondratko, P. T., Greenhill, L. J., \& Moran, J. M. 2005, ApJ, 618, 618

Krolik, J. H., \& Begelman, M. C. 1988, ApJ, 329, 702

Lodato, G., \& Bertin, G. 2003, A\&A, 398, 517

McMullin, J. P., Waters, B., Schiebel, D., Young, W., \& Golap, K. 2007, in ASP Conf. Ser. 376, Astronomical Data Analysis Software and Systems XVI, ed. R. A. Shaw, F. Hill, \& D. J. Bell, (San Francisco, CA: ASP), 127

Müller Sánchez, F., Davies, R. I., Genzel, R., et al. 2009, ApJ, 691, 749

Nenkova, M., Sirocky, M. M., Nikutta, R., Ivezić, Ž., \& Elitzur, M. 2008, ApJ, 685,160

Pestalozzi, M. R., Elitzur, M., \& Conway, J. E. 2009, A\&A, 501, 999

Savitzky, A., \& Golay, M. J. E. 1964, AnaCh, 38, 1627

Urry, C. M., \& Padovani, P. 1995, PASP, 107, 803

Vazquez, B., Galianni, P., Richmond, M., et al. 2015, ApJ, 801, 127 